\title{
An eye fixation analysis of choice and judgment with multiattribute stimuli
}

\author{
LARRY D. ROSEN and PAUL ROSENKOETTER \\ University of California, San Diego, La Jolla, Califormia 92057
}

\begin{abstract}
The present paper examined the selection of processing heuristics for choice and judgment across a range of stimuli. Eye fixations were monitored while six subjects made choices or judgments of pairs of gambles, vacations, or gifts. Each stimulus was represented by three attributes that varied in the amount of dimensional interdependency. The two heuristics of interest were dimensional and holistic evaluation. Each manifested a characteristic pattern of eye fixations. Dimensional evaluation required alternating fixations from a single attribute of one stimulus to the same attribute of the other stimulus. Holistic evaluation was characterized by transitions from attribute to attribute within a single stimulus. The results demonstrated that when the stimulus attributes were either interdependent (gambles) or dissimilar (gifts), the processing heuristic was determined by stimulus characteristics. When the stimulus dimensions were neither interdependent nor dissimilar (vacations), the selection of a processing strategy was determined by the prescribed task. This study suggests that any global theory of choice or judgment must be validated over a wide range of stimuli.
\end{abstract}

In this paper we will investigate strategies that are used to assimilate multiattribute stimulus information into an absolute or relative judgment. Specifically, we will examine two strategies, dimensional and holistic evaluation, across tasks and different multiattribute stimuli.

Although decision making and judgment have been studied extensively, no attempt has been made to compare and relate their respective processes. In the most comprehensive recent review of these fields, Slovic and Lichenstein (1971, p. 652) state that "the distinction between judgments and decisions is a tenuous one and will not be maintained here; we shall use these terms interchangeably." In this research we used an eye fixation analysis to distinguish between choice and judgment at a process or strategy level. To this end, eye fixations were monitored while subjects chose between, or judged the attractiveness of, pairs of multiattribute stimuli. The stimulus pairs were selected to represent a range of attribute (or dimensional) configurations from interdependent to dissimilar.

In the following sections we will elaborate on judgment processes, choice processes, attribute configurations and strategy selection, and eye fixation monitoring. With this background, we will test process predictions based on eye fixation measures.

\section{Judgment Processes}

Typically, the investigation of judgment processes

This research was partially supported by Grant MH- 15828 from the National Institute of Mental Health to the Center for Human Information Processing, University of California, San Diego. Requests for reprints should be sent to Dr. Larry Rosen, Psychology Department, California State College, Dominguez Hills, California 90747. The second author is currently at University of California, Los Angeles, School of Management. has required subjects to rate the attractiveness of a multiattribute stimulus. Substantial research efforts have examined two-outcome gambles (Slovic \& Lichtenstein, 1968), judgments of personality (Anderson, 1962, 1968), judgments of performance in college (Dawes, 1971), psychophysical scaling (Anderson, 1970), probability estimation (Phillips \& Edwards, 1966), clinical appraisal (Goldberg, 1965), medical diagnosis (Hammond, 1955; Hoffman, Slovic, \& Rorer, 1968), and judgments in uncertain environments (Brunswik, 1956). The typical judgment model depicts man as an algebraic integrator of information who first evaluates attributes separately and then combines the valuations according to some simple rule. For example, Anderson (1965) demonstrated that likeableness impressions of a person described by a pair of adjectives were formed by combining the attractiveness of each descriptor in a weighted average. This strategy is called holistic evaluation.

\section{Decision Processes}

Decision processes are more difficult to identify. This is not to say that the processes themselves are more complex. Rather, empirical studies of decisions have been largely ignored in favor of more theoretical pursuits. Unfortunately, these models have rarely proposed processing heuristics. One exception is Tversky's (1972) "elimination-by-aspects" choice model. In this model, each choice alternative is represented by dimensions or aspects. Applying an elimination-byaspects choice strategy, a single dimension is sampled and all of the alternatives below some criterion are discarded. This strategy is applied recursively until only one alternative remains.

Elimination by aspects is a dimensional strategy. Unlike the holistic strategy used in judgment, dimen- 
sional evaluations do not encompass the entire stimulus. Instead, comparable attributes of the choice set are evaluated and these dimensional evaluations are integrated into a final decision. Support for dimensional choice strategies has been presented by Payne (1975), Russo and Rosen (1975), Russo and Dosher (Note 1), and Svenson (Note 2).

\section{Attribute Configuration and Strategy Selection}

The prescribed task may not necessarily determine the selection of a processing strategy. For instance, although it is natural to match a holistic strategy with judgment and a dimensional strategy with choice, this assignment is by no means compulsory. Consider a choice between the following pair of gambles. Each gamble presents an amount of money to be won (e.g., $\$ 4.29$ ), a probability of winning (.44), an amount to be lost (\$1.29), and an implied chance of losing (.56). Using a straightforward dimensional evaluation strategy, the two gambles would first be compared on each attribute and then these dimensional evaluations would be combined.

$\begin{array}{cc}\text { Gamble A } & \text { Gamble B } \\ +\$ 4.29 & +\$ 2.85 \\ .44 & .72 \\ -\$ 1.29 & -\$ 2.80\end{array}$

In the example above, the payoff in Gamble $A$ exceeds that of Gamble B by $\$ 1.44$. Similarly, the probability in Gamble B exceeds that in Gamble A by .28. At this point, the dimensional evaluation scheme begins to disintegrate. The knowledge about the probability cannot easily be evaluated in the absence of information about the corresponding payoffs. The attributes of each gamble are not independent. As demonstrated here, it is difficult to concatenate such dimensional evaluations. Thus, a dimensional evaluation heuristic may not be appropriate for all decisions.

Analogously, the selection of a judgment heuristic may also be restricted by the stimulus environment. For instance, if the stimulus attributes are highly dissimilar, a holistic evaluation may be an impractical strategy for judgment. Combining such attributes may be analogous to adding apples and oranges. The units are too dissimilar to be combined into a single evaluation.

In this study, we examine processing heuristics for choice and judgment across stimuli that vary in dimensional dependence. The most dimensionally interdependent stimuli presented were gambles, similar to the example presented earlier. The least dependent, or most dissimilar, stimulus was a gift package. Each package included three attributes that were selected by independent judges as the most dissimilar. The third stimulus, a vacation package, represented an attribute configuration whose dimensions were less interdependent than the gamble and more interdependent than the gift package.
In summary, although judgment is often associated with holistic evaluation and choice with dimensional evaluation, this correspondence may depend upon the composition of the stimulus environment. With stimuli whose attributes are either interdependent or highly dissimilar, the selection of a processing strategy may be restricted. This research examines the selection of processing strategies as a dual function of the prescribed task and the stimulus environment.

\section{Eye Fixation Monitoring}

An eye fixation sequence provides one processtracing protocol for differentiating between information processing strategies. The use of eye fixations in this research differs from the ordinary use of such data. Typically, the data collected from an eye fixation sequence are collapsed into summary measures like direction of gaze (Bakan \& Shotland, 1969) or fixation density (Mackworth \& Morandi, 1967). In complex tasks, however, the strategies under investigation may incorporate more than a single eye fixation. In particular, the two strategies of interest, holistic and dimensional evaluation, each manifest a characteristic pattern of eye fixations. For example, if a dimensional evaluation strategy is employed, the eye fixation sequence should exhibit an alternating series of fixations from a single dimension of one stimulus alternative to the same dimension of the other stimulus. Correspondingly, if a holistic evaluation strategy is used, the eye fixations should travel from dimension to dimension within a single alternative.

For the present investigation only single-step eye fixation transitions will be considered. Because only two stimulus alternatives were presented for each task, a dimensional evaluation could involve at most two pieces of information, one from each alternative. With this limitation on the stimulus environment, it was assumed that two eye fixations would be sufficient to encode and evaluate a single attribute between two stimulus alternatives. As corroboration, Russo and Dosher (Note 1) found single transition measures superior to multiple transition measures for identifying binary choice strategies.

An eye fixation protocol contains two types of singlestep fixation transitions, intradimensional and interdimensional. The former may be further partitioned into transitions within a common dimension (e.g., from the probability in Gamble $A$ to the probability in Gamble B) or transitions from one dimension to another (e.g., from the probability in Gamble A to the payoff in Gamble B). The common dimension, intradimensional transitions were those suggested by a dimensional strategy. The remaining intradimensional transitions, termed diagonal transitions, were difficult to interpret and, thus, were analyzed separately.

Although either intradimensional transitions (repre- 
senting a dimensional strategy) or interdimensional transitions (representing a holistic strategy) could have been chosen as the subsequence of interest, only the former will be presented to avoid redundancy. The pattern of results was the same with both sequences.

\section{Predictions}

With the preceding discussion as background, we make the following predictions. First, choice will generally be performed with a dimensional evaluation heuristic and judgment with a holistic evaluation strategy. Second, if the dimensions of the stimuli are interdependent, a holistic strategy may be selected regardless, of the task. Third, if the dimensions are dissimilar, a dimensional heuristic may be used.

To test these predictions, subjects were required to perform three tasks (choice, judgment, and strength of preference) on three types of stimuli (gambles, vacations, and gift packages). These stimuli were selected to represent a wide range of interdimensional dependency from heavily interdependent (gambles) to essentially independent or dissimilar (gifts). The strength-ofpreference task was included to examine strategy selection for a task subsuming both a choice process and a judgment process.

\section{METHOD}

\section{Stimuli}

Each stimulus consisted of two three-attribute alternatives. All stimuli were displayed in a 3 by 2 rectangular array on a storage CRT (Model 611, Tektronix) positioned about $40 \mathrm{~cm}$ in front of the subject. The entire stimulus subtended visual angles of $8 \mathrm{deg}$ horizontally and $20 \mathrm{deg}$ vertically, with a minimum separation between dimensions of 5.5 deg. Individual alphanumeric characters were $.5 \mathrm{deg}$ high.

The three levels of dimensional dependency were embodied by three types of stimuli: gambles, vacations, and gifts. The most dimensionally interdependent stimuli were gambles. Each gamble presented a payoff, a loss, and a probability of winning. The complementary probability of losing was not visually presented. Both the payoff and loss varied between $\$ 1$ and $\$ 10$.

Gift packages represented stimuli with independent or dissimilar attributes. Each gift package included tickets for entertainment ( 1 to 5 pairs), gallons of gasoline (40 to 200), and time in a supermarket to collect food items ( 30 to $240 \mathrm{sec}$ ). In a pretest, these gifts were selected from among a group of 15 as the most dissimilar. The intervals were chosen to equate the range of dollar values across at tributes.

The third stimulus was a vacation package. This stimulus contained attributes that were less interdependent than gambles and more interdependent than the gift packages. Each vacation package specified a country, a period of time to visit that country (from 3 to 21 days), and an amount of extra spending money for the vacation ( $\$ 50$ to $\$ 500$ ). Transportation, food, and lodging were included in all vacations. In a pretest, the dimensions of each vacation were judged to be neither extremely interdependent nor dissimilar by a group of independent judges.

\section{Utility Measurement}

Prior to the eye fixation collection, each subject rated samples of the three types of stimuli. Each sample included 50 individual stimuli, that is, halves of the experimental stimulus pairs, and each sample was rated (independently) on a 100-point scale. A linear model was used to estimate dimensional weights from these single stimulus ratings. These weights served two purposes. First, they provided a test that all dimensions of each stimulus were being considered in the evaluation. By this standard, no subject ignored any dimension of any stimulus. Second, the derived attribute weights were used to generate experimental stimuli whose utilities (ratings) were approxinately equal both across subjects and within a single subject. No attempt was made to assess the appropriateness of the linear model. Rather, it was used as a convenient method for estimating subjective utilities without requiring ratings of all experimental stimuli.

\section{Design}

The design was a 3 by 3 by 3 factorial, with three tasks, three stimuli, and three levels of utility differences between the stimuli in each stimulus pair. The three tasks were: (1) preferential choice between the stimulus pairs, (2) judgment of the stimulus pair, and (3) preferential choice followed by a strengthof-preference judgment. For half of the subjects the choice task was followed by the judgment task. All subjects performed the strength-of-preference task last.

The three stimulus classes were: (1) gambles, (2) vacations, and (3) gifts. Within any task the different stimuli were presented in a Latin square design that minimized order effects (Winer, 1971).

From previous pilot work, three levels of utility differences were chosen, labeled high (greater than 5 units), medium (between 1.5 and 5 units apart), and low (less than 1.5 units apart). For each of the nine experimental conditions (tasks by stimuli), stimulus pairs were randomly generated to include 8 pairs with high, 16 with medium, and 16 with low utility differences. No attribute value was duplicated within a single experimental pair. Also, within any stimulus pair, no alternative was superior on all three attributes.

In all, 360 experimental trials were presented to each subject, 40 stimulus pairs in each of the nine task by stimulus conditions. In addition, five practice trials preceded each set of experimental trials.

\section{Eye Fixation Analysis}

The eye position was detected by a photoelectric sensing apparatus (Russo \& Mathews, Note 3). In order to restrict head movements, the subject's head was positioned on a bite bar during the task.

Eye positions were sensed 100 times/sec and recorded by a PDP-12 digtal computer (Digital Equipment Corporation). The individual eye position coordinates were collapsed into a sequence of eye fixations by an algorithm based on a pretrial calibration and a minimum fixation duration criterion of 200 msec (Goode \& Russo, 1970). During a trial the subject's eye position and calibration boundaries were displayed to the experimenter who monitored the data. This on-line monitoring procedure, combined with the wide separation between possible fixation points, minimized the chance of error in the recorded sequence of visual fixations. Trials containing errors (e.g., subject looked outside stimulus array) were rerun at the end of the experimental condition.

The following analysis was performed on single-step eye fixation transitions. The proportion of transitions for the nine task by stimulus conditions were computed for each subject. The sample sizes for these proportions ranged from a minimum of 89 eye fixations to a maximum of 1,036 (median $=372$ ). In collapsing over subjects, each proportion was combined in an unweighted average.

The initial transition to each attribute was not included in this analysis. This decision was based on evidence presented by Russo and Dosher (Note 1) on the binary choice process. Their prompted verbal protocols showed that no evaluative processing had taken place during the first eye fixations. Rather, these transitions reflected individual reading patterns. Based on this result, initial eye fixations were analyzed separately.

A preliminary analysis demonstrated no effect of utility 
difference on the amount of dimensional processing. This suggests that the subjects processed easy choices (large utility differences) in the same manner as difficult choices (small utility differences). In all analyses the proportions of dimensional transitions were collapsed over all levels of utility difference.

\section{Subjects}

Six naive student volunteers, two males and four females, participated in all conditions of the experiment. The utility collection provided every subject with some experience with both the stimuli and the tasks, prior to the recording of eye fixation protocols. All subjects were paid $\$ 1.88 / \mathrm{h}$ for their participation.

Subjects were selected on two criteria. First, the eye position apparatus required that the sclera be visible below the iris and above the eyelid while the subject was viewing straight ahead. Individuals who wore glasses were excluded. Second, each dimension in the linear utility model was required to account for more than $2 \%$ of the variance. No subject failed to meet either criterion.

\section{Procedure}

Prior to the eye fixation monitoring, subjects performed two preparatory tasks. First, each subject rated the 150 single stimuli required for the utility estimation. Second, to familiarize the subject with the experimental stimuli, sample stimulus pairs were presented for a paper-and-pencil preferential choice.

Each subject participated in four to six sessions of not more than $2 \mathrm{~h}$ each. The first session included five practice sets to familiarize the subject with the eye fixation apparatus. In order to minimize changes in utilities over time, all sessions were completed within a period of 1 week.

\section{RESULTS}

\section{Dimensional Processing}

Over all tasks the amount of dimensional processing increased from gambles (.334) to vacations (.356) to gifts $(.471)$. These differences were statistically significant according to an analysis of variance performed on the arc sine transformed proportions $[\mathrm{F}(2,10)=9.85$, $\mathrm{p}<.01 ; \mathrm{MSe}=.634]$. For individual subjects, 7 of a possible 18 conditions ( 6 subjects by 3 tasks) exhibited an increase in dimensional processing from gambles to vacations to gifts. Of the remaining conditions, eight showed only a reversal between gambles and vacations. Thus, for $83.3 \%$ of the conditions, the proportion of dimensional transitions for both gambles and vacations was less than that for gifts.

The magnitude of these proportions must be compared to the proportions of holistic transitions to draw conclusions about the strategy preference; therefore, the proportions of diagonal transitions were deleted from the analysis. For the stimuli, the proportion of diagonal transitions were: gambles, .121; vacations, .128; and gifts, .142. Removing these fixations and recalculating the proportions for the remaining transitions yielded the following proportions: gambles, .380 (dimensional) and .620 (holistic); vacations, .408 (dimensional) and .592 (holistic); gifts, .549 (dimensional) and .451 (holistic). Thus, for gambles and vacations, holistic processing was the dominant strategy. For gifts, however, a dimensional strategy was used more often,

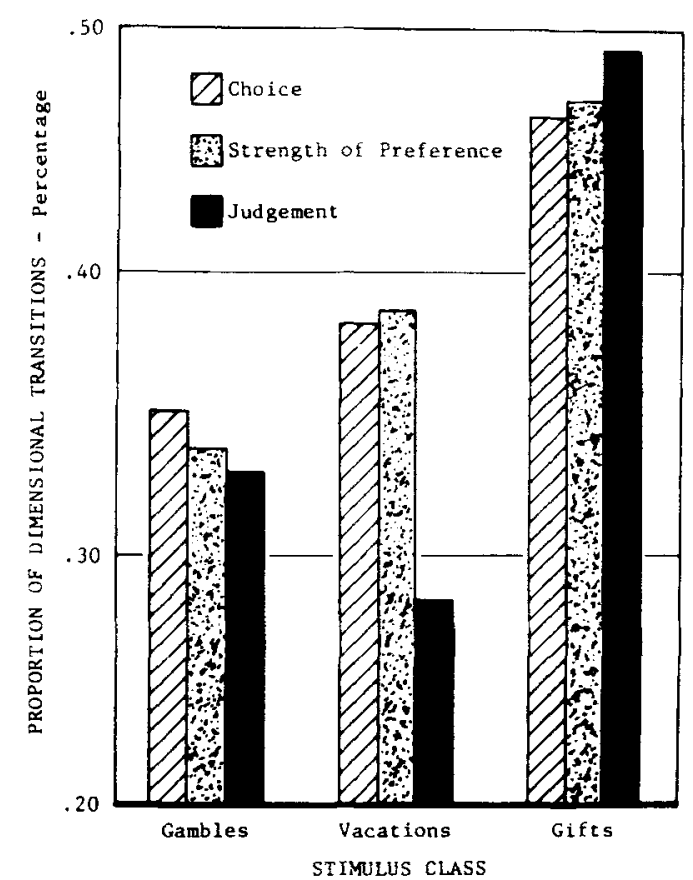

Figure 1. Proportion of postreading dimensional transitions as a dual function of task and stimulus.

although the margin of difference was nearly half that of the gambles and vacations.

\section{Processing Strategies}

Three predictions were made concerning the choice of processing heuristics across stimulus and task environments. First, if the stimulus attributes are interdependent, a holistic strategy may be used regardless of the task. The reanalysis of dimensional and holistic transitions provides partial support for this prediction. Over all tasks, holistic transitions exceeded dimensional transitions .620 to .380 . Further corroborative data are presented in Figure 1. This figure depicts the proportion of dimensional transitions (out of all transitions) for the nine experimental conditions. From inspection, it is clear that the data for the choice and the strength-of-perference tasks are remarkably similar. All further comparisons will consider only choice vs. judgment.

To assess the difference in dimensional processing for gambles, planned comparisons were performed between the proportion of dimensional transitions for choice and judgment. For completeness, two planned comparisons were performed. The liberal test (Winer, 1971, pp. 269-271) uses the Task by Stimulus interaction as the error term. The conservative test (Keppel, 1973 , p. 409) computes the error term from the withincell variability for the cells in question. No significant differences were found between the proportion of dimensional transitions for gambles (choice, . 347 vs. judgment, .323) with either the conservative or liberal test. Thus, a holistic strategy was used for interdependent stimuli for both choice and judgment. 
The second prediction stated that, if the attributes are dissimilar, a dimensional heuristic should be selected for all tasks. Considering only the two fixation sequences of interest, dimensional transitions exceeded holistic transitions .549 to .451 . From the data presented in Figure 1, no significant differences were found between the proportion of dimensional transitions for gifts (choice, $.457 \mathrm{v}$. judgment, .487) with either the conservative or liberal planned comparisons. This suggests that a dimensional strategy was used for stimuli with dissimilar attributes for both choice and judgment.

The final prediction stated that, when the stimulus environment presented less extreme attribute configurations, choice would be performed with a dimensional strategy and judgment with a holistic strategy. Vacation packages represented such a stimulus environment. In contrast with the results for the other stimulus configurations, a greater number of dimensional transitions were required to choose between two vacations $(.380)$ than to judge the same pair (.288). This difference was significant by the liberal planned comparison $[F(1,20)=6.46, p<.02]$ and marginally significant by the conservative test $[F(1,5)=5.73, p<.07]$.

The second half of the prediction was not validated. When the proportions of dimensional and holistic transitions were recomputed with the diagonal transitions removed, both choice (dimensional, .431; holistic, .569 ) and judgment (dimensional, .331; holistic, .669) required more holistic than dimensional transitions. As expected from the previous result, however, the holistic margin for judgment was decidedly greater than that for choice.

This pattern of results, task-independent strategies for gambles and gifts, and task-dependent strategies for vacations, held for five of the six subjects. Subject 1 did not satisfy the first two predictions.

\section{Patterns of Information Acquisition}

An information acquisition transition was defined as the initial excursion to a single stimulus attribute. Averaged over all tasks, the proportion of dimensional reading transitions increased from gambles $(.239)$ to vacations (.316) to gifts $(.391)$. This difference was marginally significant $[F(2,10)=3.48, \quad p<.075$; MSe $=.742]$. The task, task by stimulus, and individual planned comparisons did not approach significance (all Fs $<1$ ).

Upon closer examination, it was evident that the subjects formed two distinct information acquisition groups. Subjects 1, 2, 3, and 4 required an average of only 2.5 dimensional transitions out of every 10 eye fixations to read the stimulus pair, while Subjects 5 and 6 required an average of 4.5 . The mean proportions for each group are presented in Table 1. Note that the proportion of dimensional transitions for any stimulus for the former group was greater than the largest
Table 1

Proportion of Dimensional Reading Transitions by Dimensional and Holistic Readers

\begin{tabular}{lcccc}
\hline & \multicolumn{3}{c}{ Stimulus Class } \\
\cline { 2 - 5 } Subject Group & Gambles & Vacations & Gifts & Mean \\
\hline Holistic* & .166 & .241 & .342 & .249 \\
Dimensional** & .387 & .465 & .489 & .447 \\
Mean Over All Subjects & .239 & .316 & .391 & \\
\hline *Subjects 1, 2, 3, and 4 & & \multicolumn{4}{c}{ * *Subjects 5 and 6 }
\end{tabular}

proportion of dimensional processing for the latter group.

When the diagonal transitions are removed from the calculations, it is clear that the larger group acquired information holistically (dimensional, .286; holistic, .714 ), while the smaller group proceeded dimensionally (dimensional, .514; holistic, .486).

These global acquisition heuristics were not algorithmic. The data in Table 1 demonstrate that the amount of stimulus interdependency exerted a systematic influence on the amount of dimensional acquisition processing. This influence, however, only affected the amount of dimensional processing over a limited range, dictated by the global acquisition strategy.

\section{DISCUSSION}

The major result of this study was that the stimulus environment may have a pronounced effect on the selection of information processing strategies. When the stimulus dimensions were at the extremes of the dependency continuum, the processing heuristic was determined by stimulus characteristics. Only when the stimulus dimensions were neither interdependent nor dissimilar was the choice of a processing strategy influenced by the prescribed task.

This result may have implications for an information processing theory of choice or judgment. Any global theory must be tested on a wide range of stimuli to establish its validity. Thus far, most theories have examined only a limited stimulus domain, many restricted to the study of gambles only. An exception is the work of Norman Anderson and his associates (Anderson, 1974), whose information integration theory of human judgment has been tested on a wide range of stimuli. Unfortunately, comparable comprehensiveness has not been attempted in models of decision making.

The information acquisition fixation patterns presented a different picture. Subjects manifested two distinct information acquisition strategies. The use of each strategy was affected by the stimulus configuration, but not by the task. This suggests that a complete theory of choice or judgment should separate the information acquisition process from the remainder of the cognitive activity. 


\section{REFERENCE NOTES}

1. Russo, J. E., \& Dosher, B. A. Dimensional evaluation: $A$ heuristic for binary choice. Unpublished manuscript, University of California, San Diego, 1975.

2. Svenson, O. Coded think aloud protocols obtained when making a choice to purchase one of seven hypothetically offered houses: Some examples. Progress Report on Project of Cognitive Processing and Decision Making, University of Stockholm, 1974.

3. Russo, J. E., \& Mathews, P. A photoelectric eye position sensor. Unpublished manuscript, University of California, San Diego, 1975.

\section{REFERENCES}

Anderson, N. H. Application of an additive model to impression formation. Science, 1962, 138, 817-818.

ANDERson, N. H. Averaging versus adding as a stimuluscombination rule in impression formation. Journal of Experimental Psychology, 1965, 70, 394-400.

ANDERSON, N. H. A simple model for information integration. In R. P. Abelson, E. Aronson, W. J. McGuire, T. M. Newcomb, M. J. Rosenberg, \& P. H. Tannenbaum (Eds.), Theories of cognitive consistency: $A$ sourcebook. Chicago: Rand McNally, 1968.

ANDERSON, N. H, Functional measurement and psychophysical judgement. Psychological Review, 1970, 77, 153-170.

ANDERson, N. H. Information integration theory: A brief survey. In D. H. Krantz, R. C. Atkinson, R. D. Luce, \& P. Suppes (Eds.), Contemporary developments in mathematical psychology. San Francisco: Freeman, 1974.

Bakan, P., \& Shothand, R. L. Lateral eye movements, reading speed, and visual attention. Psychonomic Science. 1969, 15, 93-94.

Brunswik, E. Perception and the representative design of experiments. Berkeley: University of California Press, 1956.

DAWEs, R. M. A case study of graduate admissions: Application of three principles of human decision making. American Psychologist, 1971, 26, 180-188.

Goldberg, L. R. Diagnosticians versus diagnostic signs: The diagnosis of psychosis versus neurosis from the MMPI. Psychological Monographs, 1965, 79(9, Whole No. 602).

GoodE, F. M., \& Russo, J. E. A computerized system for collecting eye movement data. Proceedings of the 70th Annual Convention of the American Psychological Association, 1970, 845-846.

Hammond, K. R. Probabilistic functioning and the clinical method. Psychological Review, 1955, 62, 255-262.

Hoffman, P. J., Slovic, P., \& Rorer, L. G. An analysis-ofvariance model for the assessment of configural cue utilization in clinical judgement. Psychological Bulletin, 1968, 69, 338-349.

KEPPEL, G. Design and analysis: $A$ researcher's handbook. New York: Prentice-Hall, 1973.

Mackworti, N. H., \& Morandi, A. J. The gaze selects informative details within pictures. Perception \& Psychophysics, 1967, 2, 547-552.

PAYNe, J. W. Task complexity and contingent processing in decision making: An information search and protocol analysis. Organizational Behavior and Human Performance, 1975 , in press.

Phillips, L. D., \& Edwards, W. Conservatism in a simple probability inference task. Journal of Experimental Psychology, 1966, 72, 346-357.

Russo, J. E., \& Rosen, L. D. An eye fixation analysis of multialternative choice. Memory \& Cognition, 1975, 3, 267.276 .

Slovic, P., \& Lichtenstein, S. C. The relative importance of probabilities and payoffs in risk taking. Journal of Experimental Psychology Monograph Supplement, 1968. 78(No. 3, Part 2).

Slovic, P., \& Lichtenstein, S. C. Comparison of Bayesian and regression approaches to the study of information processing behavior in judgement. Organizational Behavior and Human Performance, 1971, 6, 649-744.

TVERSKY, A. Elimination by aspects: A theory of choice. Psychological Review, 1972, 79, 281-299.

WINER, B. J. Statistical principles in experimental design. New York: McGraw-Hill, 1971

(Received for publication November 21, 1975; revision accepted May 6, 1976.) 